\title{
THE STABLE STRUCTURE OF QUITE GENERAL LINEAR GROUPS
}

\author{
BY HYMAN BASS \\ Communicated by R. Bott, December 28, 1963
}

1. Introduction. Dieudonné [5] determined the normal subgroups of $\mathrm{GL}(n, A)$ for an (even noncommutative) field, $A$, and Klingenberg recently showed $[7 ; 8]$ that Dieudonné's result, suitably formulated, survives without surprises for $A$ any local ring. The results described here constitute the beginnings of a global theory. The information they yield on $\mathrm{SL}(n, \boldsymbol{Z})$, combined with a rather formidable cohomological calculation, is the basis of the proof in [3] that every subgroup of finite index in $\mathrm{SL}(n, Z), n \geqq 3$, contains a congruence subgroup.

This material, the details of which will appear in [1], is based on the algebraic $K$-theory described in [4]. The topological intuition thereby afforded intervenes via the space, $X$, of maximal ideals of a commutative ring, $A$. Thus, our results on $\operatorname{GL}(n, A)$ are effective only if $n$ is sufficiently large compared with $\operatorname{dim} X$, i.e. only if $n$ is in the stable range. If $A$ is semi-local this is no restriction, $X$ being then finite. If $A=Z$ then $\operatorname{dim} X=1$. For general $A$ we must let $n$ go to infinity (stabilize) ( $\$ 2$ ). While the Dieudonné-Klingenberg theorem may fail even then, its failure is measured by certain abelian groups, $K^{1}(A, \mathfrak{q})$, one for each ideal $\mathfrak{q}$. When $\mathfrak{q}=A$ we write $K^{1}(A)=K^{1}(A, A)$. When $\operatorname{dim} X=0$ they reduce to something essentially trivial, and we recover Dieudonné-Klingenberg.

In a joint paper with $\mathrm{A}$. Heller and $\mathrm{R}$. Swan [2] the homomorphisms $K^{1}(A) \rightarrow K^{1}(A[t])$ and $K^{1}(A) \rightarrow K^{1}\left(A\left[t, t^{-1}\right]\right), t$ an indeterminate, are analyzed (see \$5). Concerning the latter Atiyah has pointed out that our result is an analogue of Bott periodicity for the unitary group (see §6).

Finally, various of these results yield information ( $\$ 7)$ on J. H. C. Whitehead's groups of simple homotopy types [9], results which extend some earlier work of G. Higman [6].

2. Stable structure theorem. For a ring $A, \mathrm{E}(n, A)$ denotes the subgroup of $\mathrm{GL}(n, A)$ generated by all elementary matrices, i.e. those differing from the identity in a single, off diagonal, coordinate. If $\mathfrak{q}$ is an ideal we write

$$
\operatorname{GL}(n, A, \mathfrak{q})=\operatorname{ker}(\mathrm{GL}(n, A) \rightarrow \operatorname{GL}(n, A / \mathfrak{q})),
$$

and $\mathrm{E}(n, A, \mathrm{q})$ denotes the normal subgroup of $\mathrm{E}(n, A)$ generated by the elementary matrices in $\mathrm{GL}(n, A, \mathfrak{q})$. 
We further identify $\alpha \in \mathrm{GL}(n, A)$ with

$$
\left(\begin{array}{ll}
\alpha & 0 \\
0 & 1
\end{array}\right) \in \mathrm{GL}(n+1, A),
$$

and thus write $\mathrm{GL}(A, \mathfrak{q})=\mathrm{U}_{n} \mathrm{GL}(n, A, \mathfrak{q})$ and $\mathrm{E}(A, \mathfrak{q})=\mathrm{U}_{n} \mathrm{E}(n, A, \mathfrak{q})$. When $\mathfrak{q}=A$ we abbreviate, $\mathrm{GL}(A)=\mathrm{GL}(A, A)$ and $\mathrm{E}(A)=\mathrm{E}(A, A)$.

THEOREM. Let $A$ be any ring.

(a) For all ideals, $\mathfrak{q}$,

$$
\mathrm{E}(A, \mathfrak{q})=[\mathrm{E}(A), \mathrm{E}(A, \mathfrak{q})]=[\mathrm{GL}(A), \mathrm{GL}(A, \mathfrak{q})] .
$$

(b) If $H \subset \mathrm{GL}(A)$ is normalized by $\mathrm{E}(A)$ then for a unique ideal, $\mathfrak{q}, \mathrm{E}(A, \mathfrak{q}) \subset H \subset \mathrm{GL}(A, \mathfrak{q})$, and $H$ is then (by (a)) normal in $\mathrm{GL}(A)$.

This theorem tells us that a knowledge of the normal subgroups of $\mathrm{GL}(A)$ is equivalent to a determination of the abelian groups

When $\mathfrak{q}=A$

$$
K^{1}(A, \mathfrak{q})=\mathrm{GL}(A, \mathfrak{q}) / \mathrm{E}(A, \mathfrak{q}) .
$$

$$
K^{1}(A)=\mathrm{GL}(A) / \mathrm{E}(A)
$$

is just the commutator quotient of $\mathrm{GL}(A)$.

\section{The stable range.}

THEOREM. Suppose $A$ is an algebra, finitely generated as a module, over a commutative ring whose maximal ideal spectrum is a Noetherian space of dimension $d$.

For $n>d+1$ and for all ideals, $\mathrm{q}$ :

(a) $\mathrm{E}(n, A, \mathfrak{q})$ is normal in $\mathrm{GL}(n, A)$, and $\mathrm{GL}(n, A, \mathfrak{q})$ $=\mathrm{GL}(d+1, A, \mathfrak{q}) \mathrm{E}(n, A, \mathfrak{q})$.

Suppose $n>\max (d+1,2)$ :

(b) $\mathrm{E}(n, A, \mathfrak{q})=[\mathrm{E}(n, A), \mathrm{E}(n, A, \mathfrak{q})]=[\mathrm{E}(n, A), \mathrm{GL}(n, A, \mathfrak{q})]$ for all ideals, $\mathfrak{q}$.

(c) If $H \subset \mathrm{GL}(n, A)$ is normalized by $\mathrm{E}(n, A)$ then there is a unique ideal, $\mathfrak{q}$, such that $\mathrm{E}(n, A, \mathfrak{q}) \subset H$ and the image of $H$ in $\mathrm{GL}(n, A / \mathfrak{q})$ lies in the center.

For $n \geqq \max (2(d+1), 3)$, and for all ideals, $\mathfrak{q}$ :

(d) $\mathrm{E}(n, A, \mathfrak{q})=[\mathrm{GL}(n, A), \mathrm{GL}(n, A, \mathfrak{q})]$.

There are some technical inadequacies in this theorem, and one fundamental deficiency, which is best described by considering the homomorphisms,

$$
\mathrm{GL}(n, A, \mathfrak{q}) / \mathrm{E}(n, A, \mathfrak{q}) \stackrel{f_{n}}{\rightarrow} \mathrm{GL}(n+1, A, \mathfrak{q}) / \mathrm{E}(n+1, A, \mathfrak{q}) .
$$

These define a direct system of eventually abelian groups whose limit 
is $K^{1}(A, \mathfrak{q})$. Part (a) says $f_{n}$ is surjective for $n \geqq d+1$, and topological considerations suggest the

CONJECTURe. $f_{n}$ is injective for $n>d+2$. The affirmation of this conjecture, which would have a number of important applications, constitutes, when $A$ is a division algebra, the essential part of Dieudonné's theory of noncommutative determinants [5].

\section{Finiteness theorems.}

THEOREM. Let $\boldsymbol{\Sigma}$ be a semi-simple, finite-dimensional algebra over $\mathbf{Q}$ with $q$ simple factors, and suppose $R \otimes_{\Omega} \Sigma$ has $r$ simple factors. If $A$ is an order in $\Sigma$ and $\mathfrak{q}$ an ideal in $A$, then $\operatorname{ker}\left(K^{1}(A, \mathfrak{q}) \rightarrow K^{1}(\Sigma)\right)$ is finite, and $K^{1}(A, \mathfrak{q})$ is a finitely generated abelian group of rank $\leqq r-q$, with equality when $A / \mathfrak{q}$ is finite.

Theorem. Suppose $\Sigma$ above is simple, and let $\mathrm{SL}(n, A)$ denote the elements of reduced norm one in $\mathrm{GL}(n, A)$. Then center $\mathrm{SL}(n, A)$ $=$ center $\mathrm{E}(n, A)$ is finite. Moreover:

(a) For $n \geqq 3$ a normal subgroup of $\mathrm{E}(n, A)$ is either finite (and central), or of finite index.

(b) For all sufficiently large $n$ the same is true of $\mathrm{SL}(n, A)$.

Remarks. 1 . The theorem of $\S 3$ applies to $A$ here with $d=1$. Hence, the conjecture there alleges that $n \geqq 3$ suffices in (b) above.

2. For $A=\boldsymbol{Z}, \mathrm{SL}(n, \boldsymbol{Z})=\mathrm{E}(n, \boldsymbol{Z})$, since $\boldsymbol{Z}$ is euclidean.

5. Polynomial and related extensions. The results announced here are from a joint paper with A. Heller and R. Swan, [2].

For any ring $A$ we denote the Grothendieck group of finitely generated projective right $A$-modules by $K^{0}(A)$.

Theorem. Let $A$ be a ring and $t$ an indeterminate. There are natural split exact sequences,

$$
0 \rightarrow U_{0} \rightarrow K^{1}(A[t]) \rightarrow K^{1}(A) \rightarrow 0
$$

and

$$
0 \rightarrow U \rightarrow K^{1}\left(A\left[t, t^{-1}\right]\right) \rightarrow K^{0}(A) \oplus K^{1}(A) \rightarrow 0 .
$$

Here $U$ denotes the image of matrices of the form $1+\left(t^{ \pm 1}-1\right) \alpha$, with $\alpha$ nilpotent over $A$.

We call $A$ right regular if $A$ is right Noetherian and if every finitely generated right $A$-module has a finite projective resolution. By the Syzygy Theorem regularity of $A$ is inherited by $A[t]$, and hence also by its ring of quotients, $A\left[t, t^{-1}\right]$. 
THEOREM. If $A$ is right regular then all unipotents in $\mathrm{GL}(A)$ are trivial in $K^{1}(A)$.

COROLLARY. If $A$ is right regular and $t_{1}, \cdots, t_{n}$ are indeterminates then $K^{i}\left(A\left[t_{1}, \cdots, t_{n}\right]\right)=K^{i}(A), i=0,1$. (For $i=0$ this result is due to Grothendieck.)

CoROLlary. Let $\pi$ be a free abelian group of rank $n$, and let $A$ be a right regular ring. Then

$$
K^{0}(A \pi)=K^{0}(A) \text {, and } K^{1}(A \pi)=K^{0}(A)^{n}+K^{1}(A) .
$$

6. Relation to Bott's theorem. Let $X$ be a finite CW-complex, and let $A=\boldsymbol{C}(X)$, the ring of complex continuous functions on $X$. If $t: X \times S^{1} \rightarrow C$ sends $(x, z)$ to $z$ we have $A\left[t, t^{-1}\right] \subset C\left(X \times S^{1}\right)$. Let $\left(A^{*}\right)^{0}$ denote the group of functions $X \rightarrow C^{*}$ homotopic to a constant.

THEOREM. There is a commutative diagram,

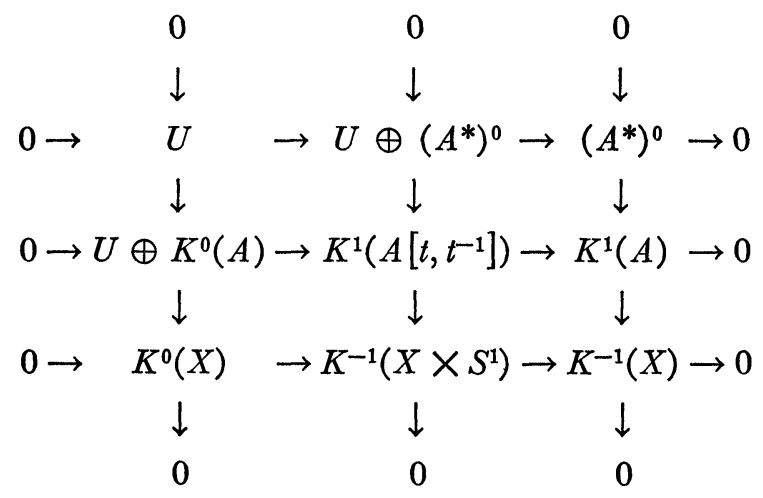

with exact rows and columns.

The middle row here comes from the theorem of $\$ 5$. Exactness of the bottom row is the unitary periodicity theorem. The ideas of the recent proof of Atiyah-Bott can be used to derive the latter from the former.

7. Groups of simple homotopy types. If $\pi$ is a group, we write Wh $(\pi)=K^{1}(\boldsymbol{Z} \pi) / \pm \pi$, meaning $K^{1}(\boldsymbol{Z} \pi)$ reduced modulo the image of $\pm \pi \subset \mathrm{GL}\left(1, \boldsymbol{Z}_{\pi}\right) \subset \mathrm{GL}\left(\boldsymbol{Z}_{\pi}\right)$. J. H. C. Whitehead's simple homotopy types [9] are topological invariants which live in these groups. From $\$ 4$ we have:

THEOREM. Let $\pi$ be a finite group with $q$ irreducible rational representations and $r$ irreducible real representations. Then $\mathrm{Wh}(\pi)$ is a finitely generated abelian group of rank $r-q$. 
It is known (Artin, Witt) that $q$ is the number of conjugacy classes of cyclic subgroups, and $r$ is the number of $\sim$ classes, where $a \sim b$ in $\pi$ means $a$ is conjugate to $b^{ \pm 1}$.

ExAmples. 1. For $\pi$ finite abelian $r=q \Leftrightarrow \pi$ has exponent 4 or 6 . C. Higman has shown [6] that $\mathrm{Wh}(\pi)=0$ if $[\pi: 1] \leqq 4$. Using the result of [3] one can show $\operatorname{Wh}(\pi)=0$ for $\pi$ of type $(2,2, \cdots)$.

2. If $Q$ is a splitting field for $\pi$ then $r=q$. This case includes the symmetric groups. $r=q$ also for the quaternion group.

3. If $\pi=\boldsymbol{Z} / n \boldsymbol{Z}$ then $q$ is the number of divisors of $n$, and $r=[n / 2]$ +1 .

From $\$ 6$ we have:

Theorem. If $\pi$ is free abelian $\mathrm{Wh}(\pi)=0$.

\section{REFERENCES}

1. H. Bass, K-theory and stable algebra, Inst. Hautes Etudes Sci. Publ. Math.

2. H. Bass, A. Heller and R. Swan, The Whitehead group of a polynomial extension, (to appear).

3. H. Bass, M. Lazard and J.-P. Serre, Sous groupes d'indice fini dans $S L(n, Z)$, Bull. Amer. Math. Soc. 70 (1964), 385-392.

4. H. Bass and S. Schanuel, The homotopy theory of projective modules, Bull. Amer. Math. Soc. 68 (1962), 425-428.

5. J. Dieudonné, Les déterminants sur un corps non commutatif, Bull. Soc. Math. France 71 (1943), 27-45.

6. G. Higman, The units of group rings, Proc. London Math. Soc. 46 (1940), 231-248.

7. W. Klingenberg, Lineare Gruppen über lokalen Ringen, Amer. J. Math. 83 (1961), 137-153.

8. - Die Struktur der linearen Gruppe ïber einem nichtkommutatives lokalen Ring, Arch. Math. 13 (1962), 73-81.

9. J. H. C. Whitehead, Simple homotopy types, Amer. J. Math. 72 (1950), 1-57

Columbia University 